\title{
SEXUALIDAD DE LOS NIÑOS, NIÑAS Y JÓVENES CON DISCAPACIDAD
}

\author{
Paola Rivera Sánchez \\ Estudiante de la Maestría en Planificación Curricular \\ Universidad de Costa Rica \\ San José, Costa Rica
}

Recibido: 27-XI-2007 • Aceptado 1-III-2008 • Corregido 3-VI-2008

\begin{abstract}
Resumen: El presente artículo es un acercamiento hacia la importancia de la vivencia de la sexualidad de los niños, niñas y jóvenes con discapacidad. Se pretende desmitificar las ideas presentes en torno a la temática de educación sexual en personas con discapacidad, asi como brindar las herramientas necesarias para que se inicie la educación sexual de ellas desde edades tempranas, con el objetivo de formar hombres y mujeres capaces de sentirse independientes para poder vivir plenamente dentro de la sociedad; sin sentirse limitados por su condición física o mental.
\end{abstract}

Palabras clave: Educación sexual, discapacidad, sexualidad, desarrollo psicosexual.

\begin{abstract}
The present article is an approach towards the importance of the experience of sexuality of children and young people with disabilities. It tries to demystify the current ideas around the theme of sexual education in people with disabilities, as well as to offer the necessary tools so that the sexual education of them begins in early ages, with the objective to form men and women able to feel independent to be able to live totally within the society; without feeling limited by its physical or mental condition.
\end{abstract}

Key words: Sexual education, disabilities, sexuality, psicosexual development.

\section{Introducción}

La sexualidad forma parte del ser humano, sin importar su condición física, social, sexo o religión; por lo tanto, no se desliga de la población infantil con algún tipo de discapacidad. Se parte del concepto que expone el Ministerio de Salud de Costa Rica, definiendo a la discapacidad como una:

(...) Condición humana que puede ser de origen multicausal, ya sea por condición genética, pobreza, desnutrición e inadecuados hábitos de salud e higiene, enfermedad o accidente laboral, deportivo, recreativo y de tránsito, contaminación ambiental, violencia política, social y estructural, etc.; no es discriminatoria por razón de género, estrato social, etnia, religión o nivel educativo o de ingresos (Ministerio de Salud. Organización Panamericana para la Salud, 2004, p. 7).

La vivencia de una sexualidad sana, permite a las personas conocerse y aceptarse a sí mismos/mismas y poder expresarla a los demás sin violentar sus derechos humanos. Para ello, es necesario que los padres, madres y docentes conozcan acerca de la importancia de la educación de la sexualidad para los niños y niñas con discapacidad, con 
el fin de contribuir a desmitificar la idea de que esta población es "asexuada" o que serán "ángeles eternos".

De esta manera, la sexualidad se convierte en un tema de interés para la población actualmente, pues al encontrarse en esta condición se vuelve doblemente discriminada por la sociedad y sus familias, debido a que se les limita expresarse libremente a través de su sexualidad.

El Código de la Niñez y la Adolescencia (1997, s.f. Capítulo IV Derecho a la Salud, parr. 35), establece que:

Artículo $55^{\circ}$ - Obligaciones de autoridades educativas. Será obligación de los directores, representantes legales o encargados de los centros de enseñanza de educación general básica preescolar, maternal u otra organización, pública o privada, de atención a las personas menores de edad: (...)

c) Poner en ejecución los programas de educación sobre salud preventiva, sexual y reproductiva que formule el ministerio del ramo (...).

Asimismo, el artículo 58 de este mismo documento, afirma que se debe propiciar la inclusión de temas relacionados con la educación sexual en los programas educativos, por lo tanto, es obligación del Ministerio de Educación Pública, poner en ejecución programas de educación sexual para la población menor de edad. Es importante tener en cuenta que lo convenido en este código no hace diferencias para la población menor de edad con o sin discapacidad.

Por otro lado, el Artículo 3.- Objetivos, de la Ley 7600 Igualdad de Oportunidades para las personas con discapacidad, presenta sus objetivos, entre ellos se pueden destacar los siguientes:

b) Garantizar la igualdad de oportunidades para la población costarricense en ámbitos como: salud, educación, trabajo, vida familiar, recreación, deportes, cultura y todos los demás ámbitos establecidos.

c) Eliminar cualquier tipo de discriminación hacia las personas con discapacidad (Asamblea Legislativa, 1996, Capítulo II. Principios Fundamentales, parr. 4 y 5).

Por lo tanto, es deber de la sociedad costarricense atender las necesidades en materia de sexualidad para poder brindar las mismas posibilidades al igual que a la niñez que no presenta ningún tipo de discapacidad.

Todas las personas tienen los mismos derechos, obligaciones y posibilidades para desarrollar todo el potencial que las personas llevan consigo, construyendo de esta manera una sociedad más justa y solidaria.

La Convención sobre los Derechos del Niño, en su artículo segundo, afirma que ningún niño puede ser discriminado por su condición física, asimismo, el artículo 23 estipula que:

...el niño mental o físicamente impedido deberá disfrutar de una vida plena y decente en condiciones que aseguren su dignidad, le permitan llegar a bastarse a sí mismo y faciliten la participación activa del niño en la comunidad (Oficina de Alto Comisionado para los Derechos Humanos. 1990, Artículo 23, parr. 1).

Partiendo de esta idea, educar y atender la sexualidad de los infantes y las infantes con algún tipo de discapacidad, les permitirá desarrollarse de manera integral, aprendiendo a conocerse, aceptarse y respetarse como hombres y mujeres que son importantes, personas a quienes se les ama tal y como son; esto les facilitará integrarse a la sociedad de manera activa.

Al conocer sobre la importancia de vivir la sexualidad a plenitud, se minimizan todos aquellos mitos que han discriminado poco a poco a esta población.

Además, reflexionar sobre la importancia de ir educando a los niños y niñas de acuerdo con su desarrollo, necesidades e intereses; evita que sean más vulnerables a abusos por parte de otras personas. Se trata de aprender a hablar del tema con naturalidad, brindándoles a los encargados y las encargadas, seguridad para apoyar a sus hijos e hijas tanto en su desarrollo físico como emocional.

Al igual que los demás niños y niñas, las personas con discapacidad exploran su cuerpo, se interesan por relacionarse con sus compañeros y compañeras, establecer 
lazos afectivos con su familia y amigos, así como saber cómo nacieron, cómo es su cuerpo y por qué éste cambia día a día. Asimismo, es necesario definir el concepto de sexualidad humana de manera integral, teniendo en cuenta los aspectos psicológicos, espirituales, culturales, físicos y sociales, de hombres y mujeres que conforman este grupo de estudio.

La educación sexual para seres con discapacidad está muy limitada en nuestro ambiente educativo, incluso considerado como un tema tabú, ya que las autoridades correspondientes no se han pronunciado al respecto ni han dado un apoyo máximo humano a esta temática.

El propósito es desmitificar ideas presentes en torno a la temática sobre educación sexual en individuos con discapacidad; y brindar las herramientas necesarias para que se inicie la educación sexual de éstas desde edades tempranas, con el objetivo de formar hombres y mujeres capaces de sentirse independientes para poder vivir plenamente dentro de la sociedad, sin sentirse limitados por su condición física o mental.

\section{Antecedentes acerca de la sexualidad de las personas con discapacidad}

La sexualidad de esta población es un tema que ha sido poco tratado por las organizaciones que la atienden, por sus docentes, sus familias y en general por la sociedad; debido a que giran en torno a ella una serie de creencias que invisibilizan la existencia de su sexualidad.

El Consejo Nacional de Rehabilitación y Educación Especial,

es el ente público rector en materia de discapacidad en Costa Rica encargado de orientar la política general en materia de rehabilitación y educación especial, en coordinación con los Ministerios de Salud, Educación Pública, Trabajo y Seguridad Social, así como la planificación, promoción, organización, creación y supervisión de programas y servicios de reha- bilitación y educación especial para personas físicas o mentalmente disminuidas, en todos los sectores del país. (Consejo Nacional de Rehabilitación y Educación Especial [CNREE], s.f., s.p.).

En conjunto con el CNREE, articulan acciones otras instituciones estatales como: la Universidad de Costa Rica (UCR), el Instituto Nacional de Seguros (INS), el Ministerio de Educación Pública (MEP), la Caja Costarricense de Seguro Social (CCSS), el Instituto Mixto de Ayuda Social (IMAS), el Instituto Nacional de Aprendizaje (INA), la Asociación de Industrias de Buena Voluntad, el Ministerio de Salud, el Ministerio de Trabajo, el Colegio de Trabajadores Sociales, y la Unión Costarricense de Cámaras y Asociaciones de la Empresa Privada.

Todas ellas trabajan en pro de la población con discapacidad en nuestro país. Sin embargo, al investigar sobre el trabajo que se ha desarrollado en este campo, se evidencia poco avance, debido a que se ha dado más énfasis al incremento y coordinación de los servicios de todo tipo de rehabilitación (física, psicosocial, educativa y profesional) y educación especial, así como al impulso en la incorporación a la vida social, en forma productiva, de este grupo de personas.

De acuerdo con las consultas realizadas, se reconoce la importancia del tema, pero existen pocas publicaciones o investigaciones de carácter científico que informen a la comunidad en este campo.

Existen trabajos de graduación realizados por los estudiantes de la Universidad de Costa Rica, en las carreras de Enfermería, Trabajo Social y Psicología, pero no desde la carrera de Educación Especial.

Por otro lado, el Ministerio de Educación y el Centro Nacional de Recursos para la Inclusión Educativa cuentan con varias publicaciones en el campo de la Educación Especial, más no en el tema de la sexualidad de las personas con discapacidad.

Recientemente, Costa Rica logró su participación en la Convención de los 
Derechos de las Personas con Discapacidad, el cual se firmó en marzo del 2007; esta convención busca asegurar que todas las personas con discapacidad puedan gozar de mejor calidad de vida desde su niñez hasta su vejez, con el fin de que ellas puedan vivir de forma independiente y participar en todos los aspectos de la vida al tener acceso a la educación, al trabajo, a la vida matrimonial y a gozar de derechos sexuales y reproductivos. La participación en esta convención es un gran avance para nuestro país en materia de educación y atención a las personas con discapacidad, y al mismo tiempo significa un gran reto para poder responder a sus necesidades.

\section{La sexualidad de los niños, niñas y jóvenes con discapacidad}

Antes de hablar de la sexualidad de las personas con discapacidad, es necesario definir los conceptos de discapacidad y sexualidad. A pesar de que existen múltiples acepciones para definirlas se tomarán las siguientes definiciones. Como discapacidad se entenderá:

(...) Condición humana que puede ser de origen multicausal, ya sea por condición genética, pobreza, desnutrición e inadecuados hábitos de salud e higiene, enfermedad o accidente laboral, deportivo, recreativo y de tránsito, contaminación ambiental, violencia política, social y estructural, etc.; no es discriminatoria por razón de género, estrato social, etnia, religión o nivel educativo o de ingresos (Ministerio de Salud. Organización Panamericana para la Salud, 2004, p. 7).

De acuerdo con lo establecido por el Departamento de Educación Integral de la Sexualidad Humana (DEISH) del Ministerio de Educación Pública la sexualidad,

... es un elemento básico de la personalidad, un modo propio de ser, de manifestarse, de comunicarse con los otros, de sentir, de expresarse y de vivir el amor humano y es parte integrante del desarrollo de la personalidad y de su proceso educativo; en el sexo radican notas características que constituyen a las personas como hombres y mujeres en el plano biológico, psicológico, social y espiritual. Las personas menores de edad tienen el derecho a la formación e información adecuadas y oportunas sobre la dignidad, tareas y ejercicio del amor, sobre todo en el seno de la familia (Ministerio de Educación Pública, 2001, p. 6-7).

Acorde con lo anterior, la sexualidad integra una serie de aspectos que buscan el desarrollo de las personas, para que éstas puedan participar dentro de la sociedad activamente y que al mismo tiempo se proyecte en todas sus actividades en los diversos momentos de su ciclo vital.

La sexualidad es fundamental para el desarrollo y ajuste de la personalidad de cada individuo (a), por lo tanto, todas las personas la poseen y tienen la oportunidad de vivirla a su manera, -ya que cada quien tiene sus particularidades-. De esta manera, la vivencia de la sexualidad permite aceptarse a sí mismo/misma, vivir a plenitud, apropiarse de su cuerpo y tomar sus propias decisiones con respecto a él. Partiendo de esta definición podemos ver que la sexualidad no se aparta de las personas con discapacidad, sino que forma parte integrante en ellas.

Al ser la sexualidad tan importante para el desarrollo de la persona, se hace necesaria la educación sexual para toda la población, la cual ha de iniciarse desde edades tempranas sin hacer distinciones por condición social, física o mental.

La pedagoga en sexualidad Xiomara Ruiz de la Universidad Autónoma del Estado de México, destaca dos tareas primordiales de la pedagogía de la sexualidad y que son:

1. La investigación y elaboración de métodos, vías y formas concretas que contribuyan a la formación y desarrollo de la esfera psicosexual de la personalidad.

2. La capacitación de los educadores para la educación sexual de las nuevas generaciones ${ }^{7}$.

Del cumplimiento de la primera tarea depende en gran medida que la educación sexual ocupe el lugar que le corresponde como una dirección más del proceso educativo" (Ruiz, 1999, p. 121). 
Como se evidencia, la educación de la sexualidad debe dirigirse al desarrollo de la personalidad, con el objetivo de permitir a las personas la toma de decisiones con respecto a la vivencia de su sexualidad. Esta educación no debe limitarse solamente a brindar conocimientos relacionados con los aspectos biológicos, sino que debe integrar diversos puntos de vista como por ejemplo el psicológico, el higiénico, el social y el espiritual, así como los valores y comportamientos que conforman la integralidad de la sexualidad humana.

Según el material consultado, el lenguaje y la naturalidad con la que se maneje el tema favorecerán la comunicación y el respeto entre los padres, madres, hijos y docentes cuando traten esta temática. De esta manera, los niños, niñas y jóvenes aprenderán correctamente el nombre de cada parte de su cuerpo y podrán ver su sexualidad sin el morbo que gira a su alrededor, el cual ha sido creado por las falsas creencias (mitos y estereotipos) de las personas con respecto a la vivencia de su sexualidad, los cuales se deben a la falta de información y formación adecuada sobre esta temática.

Murillo y Richmond (2005), afirman que al estudiar los estereotipos asociados a la sexualidad de este grupo poblacional, se puede encontrar una clasificación de los mitos, la cual los categoriza como mitos referidos a la "asexualización", la "sobresexualización" y sobre el "temor a la reproducción de las personas con discapacidad".

Con respecto a la asexualización, se considera según estos autores que estas personas son como angelitos y por lo tanto no tienen sexualidad (Rodríguez, 2003, citado por Murillo y Richmond, 2005), afirma:

Los prejuicios relacionados con su sexualidad oscilan en los extremos de creer que por su condición no se presentan necesidades de afecto y vida sexual, son como niños o que por su sexualidad está exacerbada no tienen control (p.7).

Referente a la sobresexualización, existe la creencia de que ellos y ellas "solo piensan en lo sexual", por el simple hecho de demostrar ciertos comportamientos sexuales en lugares públicos.

$\mathrm{Y}$ finalmente acerca de la reproducción, surge la idea de transmitir genéticamente una lesión al bebé que estará por nacer.

Con el fin de ir desmitificando estas ideas, es importante comprender el desarrollo psicosexual de la humanidad. Para ello, se debe tomar en cuenta el conocimiento que los alumnos/alumnas tienen acerca de sus experiencias cotidianas y que al mismo tiempo tienen un especial significado en sus vidas. Para Ruiz (1999, p. 124), se debe tener

conocimiento del desarrollo evolutivo de la sexualidad que permita adecuar los contenidos a las características de la edad... Somos seres sexuados desde que nacemos hasta que morimos, por tanto, la sexualidad se expresa durante toda la vida pero de diferentes formas de acuerdo a la edad de cada individuo [a], la educación que haya recibido...

Una vez más, se demuestra implícitamente que la sexualidad no es sólo de las personas que no presentan discapacidad, sino que es parte integrante de la humanidad.

Según el Better Health Channel (2004) en el artículo "Sex education for children with intellectual disabilities"[La Educación Sexual para niños con discapacidad intelectual], las personas con discapacidad tienen los mismos pensamientos, deseos, actitudes, fantasías y actividades como las personas que no presentan discapacidad. Sin embargo, esta población corre un alto riesgo de ser abusada, de tener embarazos no planificados o de incrementar el riesgo de contraer infecciones de transmisión sexual, si no se les brinda una adecuada educación sexual.

Better Health Channel (2004), además, agrega que es importante que los niños y niñas tengan la oportunidad de ser educados en sexualidad, así como darles la oportunidad de explorarse, disfrutar y expresar su sexualidad de manera positiva y saludable. 
De acuerdo con Baxley y Zendell (2005) en su manual "Sexuality education for children and adolescents with developmental disabilities: an instruccional manual for parents or caregivers of and individuals with developmental disabilities" [Educación de la sexualidad para los niños y los adolescentes con inhabilidades de desarrollo: un manual del instruccional para los padres o encargados de los individuos con inhabilidades de desarrollo], la educación sexual para niños, niñas y jóvenes con algún tipo de discapacidad debe centrarse en los siguientes tópicos:

- Reconocimiento de las partes de su cuerpo, utilizando el nombre correcto de cada una de esas partes.

- Comprensión de la diferencia entre hombres y mujeres.

- Comprensión y expresión de sus cambios emocionales.

- Comprensión e importancia de la higiene personal.

- Autoestima.

- Autocontrol.

- Derechos de sí mismo y de los demás.

- Nacimiento de un bebé.

- Abuso físico y sexual.

- Infecciones de transmisión sexual.

- Afectividad, entre otros.

Al hablar de sexualidad, es importante definir algunos conceptos básicos que están relacionados y que permitirán tener una compresión clara acerca de dicha temática, entre ellos se destacarán los utilizados por el Departamento de Educación de la Sexualidad del Ministerio de Educación, en las capacitaciones denominadas Educación de la Sexualidad para Multiplicadores (2007):

- Sexo: conjunto de características biológicas que definen a los seres vivos en dos grupos: hembras y machos. Inicia en la fecundación y se prolonga a lo largo del desarrollo y la maduración (Valerio,1998 citado en MEP,
2007). En el caso de los seres humanos/las seres humanas se denominan: hombres y mujeres (MEP, DEISH, 2002 citado en MEP, 2007).

- Identidad sexual: convicción y aceptación de ser hombre o mujer, puede corresponder al sexo biológico o no (Vega, 2004 citado en MEP, 2007). Esta convicción interna puede reflejar o no la apariencia externa o el rol de género que impone la sociedad o que uno desarrolla y prefiere. Por lo general la identidad de género y la identidad sexual van de acuerdo (Diamond, 2003 citado en MEP, 2007).

- Orientación sexual: se refiere a la preferencia-atracción erótico-afectiva de una persona hacia otra persona, se define a partir de su identidad sexual. Los términos homosexual, heterosexual y bisexual son los que se utilizan para clasificar las orientaciones sexuales.

- Homosexual: se refiere a aquellas personas cuya atracción erótica-afectiva esta orientada a personas que poseen el mismo sexo.

- Heterosexual: se refiere a aquellas personas cuya atracción erótica-afectiva esta orientada a personas que poseen el otro sexo.

- Bisexual: se refiere a aquellas personas cuya atracción erótica-afectiva está orientada a personas que poseen su mismo sexo o el otro, o ambos indistintamente.

- Género: se refiere a los aspectos psicológicos, sociales y culturales que se adjudican a las personas, básicamente, de acuerdo con su sexo. Alude a los procesos de socialización de la masculinidad y feminidad, que se llevan a cabo en hombres y mujeres [WEM] (2001 citado en MEP, 2007).

- Relaciones sexuales: en tanto toda persona es un ser sexuado, relaciones sexuales se refiere a las interacciones entre seres humanas/seres humanos. 
También se le llaman relaciones humanas.

- Genitalidad: se refiere al uso y función de los órganos genitales (Valerio, 1998 citado en MEP, 2007). Específicamente refiere al contacto (roce) entre genitales (MEP-DEISH, 2004 citado en MEP, 2007).

- Coito: relación sexual genital o no, que implica penetración pene-vagina, pene-ano (MEP-DEISH, 2004 citado en MEP, 2007).

- Afectividad: capacidad de vincularse consigo mismo(a) y con los(as) demás para el autocuidado, el cuidado y el crecimiento mutuo (Quirós, 2003 citado en MEP, 2007).

Es fundamental que tanto los padres, madres, encargados, docentes y demás profesionales que trabajan con esta población manejen estos términos, para que de esta manera puedan ir comprendiendo el por qué es importante educar en sexualidad y el por qué ésta no se limita solo a la genitalidad, sino que comprende en su totalidad el desarrollo integral de la persona.

\section{Mitos y realidades acerca de la sexualidad de las personas con discapacidad}

Los artículos e investigaciones consultadas sobre la sexualidad de las personas con discapacidad evidencian la existencia de mitos que muestran la no aceptación de los derechos de las personas con discapacidad en este ámbito, limitando el desarrollo de la educación sexual dentro de sus hogares y los salones de clase.

Es por este motivo que, la educación de la sexualidad tiene la intención de transmitir, formar y promover actitudes, valores, conocimientos y habilidades, desde tempranas edades para que se pueda vivir una sexualidad responsable.

Para desmitificar el tema de la sexualidad de los niños, niñas y adolescentes con discapacidad, es necesario brindar información adecuada que permita derribar todos los mitos existentes alrededor de este tema.

Es muy importante dejar claro que no existen diferentes sexualidades, es decir que no hay una sexualidad específica para los niños/niñas, los adultos/adultas o de las personas con discapacidad; sino que la sexualidad es una sola: la sexualidad humana, la cual se manifiesta de diversas maneras según las etapas de nuestra vida. Por lo tanto, podemos concluir que todos y todas somos seres sexuados y en consecuencia, ésta forma parte integrante a lo largo de la vida.

Desde esta perspectiva, se concibe a la persona con discapacidad, como un ser sexuado con derecho a vivir su sexualidad a pesar de su condición física y/o mental.

Es así como, antes de conocer los mitos existentes en torno a la sexualidad de las personas con discapacidad, es necesario comprender cuál es su significado; de acuerdo con la Enciclopedia Libre Universal en Español,

Un mito es un relato que tiene una explicación o simbología muy profunda para una cultura. El término es utilizado a veces de manera peyorativa para referirse a creencias comunes de una cultura o religión para dar a entender que la historia es fantástica e irreal (...) (Enciclopedia Libre Universal en Español, s.f., Mito, parr. 2).

En torno a esta temática han surgido gran cantidad de mitos. Es importante analizar cada uno de ellos y contrastarlos con la realidad. Veamos en el Cuadro No. 1 algunos ejemplos citados por Silverberg, (2006) en su artículo "Sexuality and disability: Myths and facts" [Sexualidad, Discapacidad Mitos y Hechos].

Se ha creído, por ejemplo, que las personas con discapacidad intelectual son peligrosas, que no controlan sus emociones y deseos, por lo que se comportan de manera extraña al relacionarse con los demás; esta y otras creencias están muy ligadas a la ignorancia tanto de la sociedad como de las familias de estas personas. 


\section{Cuadro $\mathrm{N}^{\circ} 1$}

\begin{tabular}{|c|c|}
\hline Mito & Realidad ${ }^{1}$ \\
\hline $\begin{array}{l}\text { Las personas con discapacidad } \\
\text { no son sexuadas. }\end{array}$ & $\begin{array}{l}\text { Todos los seres humanos somos seres sexuados y por lo tanto pueden } \\
\text { expresar su sexualidad. Las personas con discapacidad han sido priva- } \\
\text { das de sus derechos sexuales en gran parte por la sociedad, probable- } \\
\text { mente porque han sido tratados como niños/niñas. Esta población puede } \\
\text { expresar su sexualidad de diversas formas al igual que el resto de la } \\
\text { población, sin embargo, esto varía de cultura a cultura. }\end{array}$ \\
\hline $\begin{array}{l}\text { Las personas con discapacidad } \\
\text { no son deseadas. }\end{array}$ & $\begin{array}{l}\text { Las sociedades se han encargado de vender la idea de que la belleza y la } \\
\text { perfección de hombres y mujeres es lo más importante, que hay que ser } \\
\text { súper modelos o al menos contar con ciertas características físicas para } \\
\text { poder atraer a las demás personas; olvidándose así del gran valor que } \\
\text { tiene la persona en lo más profundo de su ser, sus sentimientos, valores, } \\
\text { personalidad, sus deseos de superación, entre otros. }\end{array}$ \\
\hline $\begin{array}{l}\text { Hay una manera correcta y } \\
\text { una manera incorrecta de } \\
\text { tener sexo. }\end{array}$ & $\begin{array}{l}\text { El sexo }{ }^{2} \text { se tiene de diferentes maneras, de acuerdo con las individuali- } \\
\text { dades de cada persona. }\end{array}$ \\
\hline $\begin{array}{l}\text { ¿Las personas con discapaci- } \\
\text { dad pueden tener realmente } \\
\text { sexo? }\end{array}$ & $\begin{array}{l}\text { Existe la creencia de que el sexo debe tenerse solo entre personas que } \\
\text { no tienen discapacidad y que si se tiene entre personas con discapacidad } \\
\text { esto no es válido. En realidad, ellos y ellas pueden disfrutar de tener sexo } \\
\text { con sus parejas y de expresar su sexualidad sin limitaciones. }\end{array}$ \\
\hline $\begin{array}{l}\text { La gente con discapacidad } \\
\text { tiene cosas más importantes } \\
\text { en qué pensar que en el sexo. }\end{array}$ & $\begin{array}{l}\text { No es cierto que ellos/ellas tengan cosas más importantes a las cuales } \\
\text { les deben poner más atención, su sexualidad también es importante para } \\
\text { desarrollarse de manera integral. }\end{array}$ \\
\hline $\begin{array}{l}\text { La gente con discapacidad no } \\
\text { es sexualmente aventurera. }\end{array}$ & $\begin{array}{l}\text { El hecho de que se tenga discapacidad, no quiere decir que no se tengan } \\
\text { ciertas preferencias para realizar un acto íntimo, ellos o ellas pueden } \\
\text { tener deseos e ideas para poder transmitir y sentir diversas emociones } \\
\text { al vivir su sexualidad. }\end{array}$ \\
\hline $\begin{array}{l}\text { La gente que se encuentra } \\
\text { institucionalizada no debería } \\
\text { tener sexo. }\end{array}$ & $\begin{array}{l}\text { La realidad es dura, en la mayoría de las instituciones se ha perdido } \\
\text { el derecho a la intimidad, no existen cerraduras en las habitaciones ni } \\
\text { facilitan habitaciones para las parejas de estas personas, por lo que se } \\
\text { violan sus derechos sexuales, irrespetando asimismo los derechos huma- } \\
\text { nos de este grupo. }\end{array}$ \\
\hline $\begin{array}{l}\text { Las personas con discapacidad } \\
\text { nunca serán agredidos } \\
\text { sexualmente. }\end{array}$ & $\begin{array}{l}\text { Muchas veces se cree que las personas con discapacidad no corren riesgo } \\
\text { de ser agredidos sexualmente, pero la realidad es que se encuentran más } \\
\text { propensos a sufrir de esta situación, por abusos de los médicos, padres, } \\
\text { madres, docentes o especialistas en rehabilitación. Están más propensos } \\
\text { en una relación de } 2 \text { a } 10 \text { veces más que el resto de la población. }\end{array}$ \\
\hline No necesitan educación sexual. & $\begin{array}{l}\text { Todos y todas somos iguales, por lo tanto necesitamos educación sexual, } \\
\text { la ignorancia en este tema crea un obstáculo para poder comprender } \\
\text { su propia sexualidad, limitando el acceso a la poca información que se } \\
\text { brinda al respecto, esto se acentúa más con la idea de que entre más se } \\
\text { hable del tema más se promoverá el deseo sexual. }\end{array}$ \\
\hline
\end{tabular}

1 Las realidades presentes en el cuadro son parte del artículo de Silberberg y de la experiencia profesional de Paola Rivera Sánchez, autora de este artículo.

2 Para Silverberg, el término sexo es entendido como sinónimo de relaciones coitales o relaciones genitales; sin embargo, es importante aclarar que el término correcto es el indicado en la página 11 de este artículo. 
A los niños, niñas y adolescentes les interesa vivir y disfrutar de las mismas actividades que hacen los demás chicos y chicas de su edad, por lo que se sentirán atraídos por participar de fiestas, compartir deportes o por mantener relaciones interpersonales con personas del sexo opuesto, a las cuales les pueden expresar su afecto de diversas maneras; sin embargo, esto crea una alta angustia tanto a los padres, madres y educadores y educadores que se encargan de la educación de estos niños, niñas y jóvenes.

El poder conocer y analizar cada uno de los mitos existentes en torno a esta temática, permite poder comprender los riesgos a los cuales está sometido este grupo poblacional. Algunos de esos riesgos pueden ser: embarazos no deseados, abusos sexuales, infecciones de transmisión sexual, entre otros.

Es por esta situación que la educación de la sexualidad para estas personas se vuelve una herramienta fundamental para poder enseñarles como pueden vivir su sexualidad saludablemente sin ser violentados/violentadas por su condición.

Para ello es necesario que tanto los padres, madres y docentes inicien a edades tempranas la educación de la sexualidad y que ésta sea introducida en el currículo escolar.

El punto de partida de la educación sexual puede variar entre un niño/niña y otro/otra o entre un adolescente/una adolescente y otro/otra, asimismo variará entre una discapacidad y otra, debido a que cada persona cuenta con sus propias necesidades; sin embargo, hay puntos claves en los que hay consenso al iniciar la temática. Para ir desarrollando habilidades para la vida autónoma es necesario:

- $\quad$ Establecer los límites entre las actividades que se realizan en público y en privado: identificar cuáles son las partes del cuerpo, cuáles se cubren y el motivo por el que lo hacemos, cuáles son los espacios (lugares) para la privacidad y en cuáles se puede compartir con los demás de su presencia.

- Conocer la información necesaria para el autocuidado diario.

- Identificar acerca de los límites en las relaciones con los demás (familia, amigos, docentes y extraños).

- $\quad$ Planes de seguridad, para poder saber qué hacer en caso de que alguien le toca de forma indebida.

\section{Desarrollo psicosexual}

La niñez con discapacidad presenta un desarrollo psicosexual al igual que el resto de la población que no tiene discapacidad; para ello se espera que presente ciertas características acordes con su edad. De acuerdo con (The National Information Center for Children and Youth with Disabilities, 1992) [El Centro Nacional de Información de Niños y Jóvenes con Discapacidad], el desarrollo psicosexual de esta población pasa por las siguientes etapas:

Desde el nacimiento a los tres años: exploran su cuerpo para conocerlo físicamente y para conocer sus sensaciones. La fascinación por sus genitales es parte de esta etapa, por lo que las emociones que experimentan durante el baño, o al acariciar sus genitales le permiten conocerse a sí mismo/misma. En estos momentos, los padres, madres o encargados deben aprovechar para explicarles cuáles de esas actividades se hacen en público o en privado.

De los tres a los cinco años: durante este periodo se les enseñan las partes del cuerpo de hombres y mujeres, de esta forma podrán luego identificarse a sí mismos entre alguna de estas clasificaciones. Además este espacio debe aprovecharse para nombrar adecuadamente cada una de las partes del cuerpo.

De los cinco a los ocho años: a esta edad continúa la exploración de su cuerpo, pero se inicia además el interés por descubrir el cuerpo de sus pares, esto es 
natural y normal. Es necesario enfatizar en el desarrollo de habilidades sociales. Durante este tiempo es necesario estudiar las partes del cuerpo y sus funciones, las similitudes y diferencias entre hombres y mujeres, conocer como nacen los bebés, la importancia de mantener buenas relaciones sociales como la amistad, el amor, la comunicación, entre otros.

De los ocho a los once años: a esta edad algunos niños y niñas inician su preadolescencia, por lo que se da énfasis en su imagen corporal, esta edad pasa a ser una de las más importantes de su vida, al punto que se vuelven más vulnerables, donde se presentan problemas con la percepción de sí mismos.

La comprensión, la escucha y la comunicación por parte de los padres, madres o encargados de ellos/ellas se vuelve un excelente apoyo a esta edad. La preparación física y psicológica que reciban les permitirá mitigar un poco el impacto de los cambios corporales y emocionales (como la menstruación en las mujeres y los sueños mojados en los hombres). En esta etapa se deben profundizar temáticas como: la reproducción y el embarazo, la importancia del valor en la toma de decisiones, la masturbación, abstinencia, abuso sexual, físico y emocional e infecciones de transmisión sexual.

De los doce a los 18 años: durante estas edades es importante ir dándole ciertas responsabilidades para que vayan tomando sus propias decisiones; los adolescentes y las adolescentes requieren de más privacidad e independencia. Deben escoger a sus amistades e irse integrando en diversos grupos sociales con personas de diferente edad.

También se hace necesario que se continúe reafirmando lo enseñado con respecto a las actividades que se realizan en privado y en público.

La etapa de la pubertad y la adolescencia se encuentran marcadas por los sentimientos de extrema sensibilidad con respecto a su cuerpo. Un tema muy importante por tratar es la masturbación, aunque para los familiares y encargados de los jóvenes con discapacidad, este tema resulta embarazoso, es importante que sea discutido con ellos/ellas, debido a que se debe evitar que haya prácticas en lugares inapropiados.

\section{Inicio de la educación sexual para los niños, niñas y adolescentes con discapacidad}

Tal y como se ha mencionado anteriormente, la educación sexual es fundamental para el desarrollo de toda persona, por lo tanto, ésta debe iniciarse desde edades tempranas, desde que el niño/niña empieza a gatear, caminar, a asearse de manera independiente, al ingresar a la edad preescolar, al iniciar con sus primeros cuestionamientos sobre el tema o durante la adolescencia.Cada uno de los modelos que se presenten a lo largo de su desarrollo, serán fundamentales para tomarlos de ejemplo para crecer; por lo tanto, los adultos deben orientar su aprendizaje hacia espacios que le permitan vivir la expresión de su sexualidad de manera saludable, así como establecer habilidades sociales adecuadas de acuerdo a su edad.

Esto les facilitará saber resistir a la agresión y al abuso sexual, conocer sobre su cuerpo y sobre los derechos de su privacidad.

Es importante resaltar que el inicio de la educación sexual lo marcan los niños, niñas y adolescentes, por lo que las personas que se encargan de educarlos deben tener claro que se debe responder de inmediato a los cuestionamientos que ellos realicen.

De acuerdo con Bain (s.f.) del Centro de Recursos sobre Salud Sexual SunnyHill Health Centre for Children de Canadá, se deben tomar en cuenta los siguientes tópicos de acuerdo con la edad de los niños/ niñas y adolescentes, (ver Cuadro $\mathrm{N}^{\circ} 2$ ): 
Cuadro $\mathrm{N}^{\circ} 2$

\begin{tabular}{|c|c|}
\hline Rango de edades & Tópicos por desarrollar \\
\hline De 2 a 9 años & $\begin{array}{l}\text { - Diferencias entre niños y niñas. } \\
\text { - Las partes del cuerpo. } \\
\text { - Lugares y conductas públicas y privadas. } \\
\text { - Modos de reconocer y decir no ante contactos incorrectos. } \\
\text { - Cómo nacen los niños. } \\
\text { - El autoerotismo (masturbación). }\end{array}$ \\
\hline De 10 a 15 años & $\begin{array}{l}\text { - La menstruación, sueños con derrames y otros cambios que se observan en la } \\
\text { pubertad. } \\
\text { - Sentimientos sexuales. } \\
\text { - Orientación sexual. } \\
\text { - Elaborar y mantener las relaciones. }\end{array}$ \\
\hline De 16 años en adelante & $\begin{array}{l}\text { - Diferencias entre sexo y amor. } \\
\text { - Responsabilidades de las relaciones sexuales. } \\
\text { - Responsabilidades de la paternidad. } \\
\text { - Leyes y consecuencias de los contactos incorrectos con otros. }\end{array}$ \\
\hline
\end{tabular}

Cada etapa del desarrollo es importante para el crecimiento personal, por lo tanto cada una de ellas se debe respetar para ir fortaleciendo la vivencia de su sexualidad, de manera tal que los niños, niñas y adolescentes puedan ser más autónomos, con capacidad para tomar decisiones y para demostrar a la sociedad que tienen derecho a vivir sin discriminaciones.

\section{Hablar de sexualidad}

De acuerdo con Better Health Channel (2007), en su artículo "Sex education for children with intellectual disabilities: tips for parents" [Educación Sexual para niños con discapacidad intelectual: consejos para padres] existen diversas formas para hablar sobre esto, por lo tanto es importante centrar la atención en algunas de ellas:

- Tratar de expresar la información de la manera más simple posible.

- La educación sexual debe darse diariamente, tomando en cuenta las inquietudes y conocimientos de los niños, niñas y jóvenes, para ir adicionando nuevos conocimientos a lo largo de su vida.

- $\quad$ Para iniciar el tema de las diferencias de género, se puede apoyar con muñecas y muñecos para ver correctamente cada una de las partes del cuerpo.

- Las personas con discapacidad intelectual tienen problemas para comprender situaciones o temas abstractos, por lo que es necesario asistirse con videos, dibujos, libros o cualquier material didáctico que permita hacer más concreta la educación sexual.

- Tomar en cuenta la edad y desarrollo evolutivo de los niños, niñas y adolescentes para encausar la educación de acuerdo con sus necesidades.

- Realizar actividades como por ejemplo "Juego de roles" para discutir de manera práctica aspectos relacionados con la importancia de desarrollar habilidades o la asertividad.

- Asimismo, el juego de roles permite que ellos y ellas puedan comprender cuáles son las actividades que se pueden hacer en público y cuáles en privado. 
- Demostrar de ser posible con ayuda del material didáctico, cómo nacen los bebés.

- Ver a la masturbación o autoerotismo como una forma natural y saludable de expresar y explorar su sexualidad, siempre y cuando ésta se practique en privado.

\section{Reflexiones finales}

$\mathrm{Al}$ haber realizado un recorrido sobre la importancia de la educación de la sexualidad para las personas con discapacidad, se pueden extraer las siguientes reflexiones:

- Los problemas sexuales de las personas con discapacidad se derivan del prejuicio y la discriminación y no de la deficiencia individual propiamente.

- La educación sexual beneficia a esta población al incrementar habilidades sociales.

- Mejora la asertividad.

- Impulsa la independencia.

- Aprenden que la expresión de su sexualidad es su responsabilidad.

- Permite cambiar conductas para expresar adecuadamente su propia sexualidad.

- Los jóvenes con discapacidad tienen las mismas preguntas e inquietudes que aquellos que no la tienen, por lo tanto se les debe escuchar y atender su demanda de información.

- Al educar en sexualidad se deben tener en cuenta las siguientes consideraciones: ¿cómo la discapacidad del niño, niña o joven podrá afectar su desarrollo psicosexual?, ¿cómo su discapacidad puede afectar su capacidad para aprender acerca de su sexualidad?, ¿cuál información adicional requerirá para poder vivir su sexualidad a plenitud?

- Se debe utilizar un lenguaje adecuado y concreto, que inspire respeto, franqueza y libertad de expresión.
- Honestidad al hablar, que permita una comunicación positiva y respuestas basadas en la verdad.

- Los objetivos de la sexualidad deben enseñar a pensar y a reflexionar en torno al tema.

- Es importante el respeto por las diferencias, así como la igualdad en la participación de ambos sexos.

- Los profesionales en educación y las familias deben tener conocimiento acerca del desarrollo psicosexual de sus hijos e hijas, para poder adecuar el tema acorde con su edad.

- Utilizar metodología activa y medios audiovisuales para reforzar la enseñanza, de manera que capten el interés y atiendan sus necesidades.

- Para que la educación sexual tenga éxito es importante que exista una estrecha relación entre la escuela y el hogar, con el fin de que todo se relacione entre sí, para que no hayan confusiones en los jóvenes, niños y niñas.

- Al ser cada persona un ser individual e irrepetible, es necesario crear un plan que atienda cada una de las necesidades de este grupo poblacional y para ello es necesario: determinar las necesidades presentes, definir las metas, encontrar los recursos (materiales curriculares: videos, muñecos, juegos, dibujos, entre otros), desarrollar estrategias y asignar responsabilidades y finalmente, implementar y evaluar si se cumplieron las metas.

De esta manera se visualiza que la educación de la sexualidad es esencial para el desarrollo de los niños, niñas y adolescentes con discapacidad. Ésta les permite adquirir los conocimientos necesarios para interrelacionarse con los demás y las demás de manera respetuosa, responsable y solidaria. Asimismo, les facilita la toma de decisiones para establecer en un futuro sus proyectos de vida, así como prevenir situaciones de 
riesgo como el embarazo, el abuso sexual y las infecciones de transmisión sexual.

¡Todo ello permitirá personas más autónomas y felices!

\section{Referencias bibliográficas}

Asamblea Legislativa (1996, Mayo 29). Ley 7600 Igualdad de Oportunidades para las Personas con Discapacidad. En: La Gaceta Diario Oficial. Año CXVII, $N^{o}$ 102. Extraído el 27 de abril, de 2007 de

www.oit.or.cr/bidiped/legislacion/ ley7600.htm

Bain, B. (s.f). Hablar sobre la sexualidad con mi hijo. Centro de Recursos sobre Salud Sexual SunnyHill Health Centre for Children de Canadá. Extraído el 15, octubre, de 2007 de www.down21.org/educ_psc/ sexualidad/hablar_sexualidad.htm

Baxley, D y Zendell, A (2005). Sexuality education for children and adolescents with developmental disabilities: an instruccional manual for parents or caregivers of and individuals with developmental disabilities [Educación de la sexualidad para los niños y los adolescentes con inhabilidades de desarrollo: un manual del instruccional para los padres o encargados de los individuos con inhabilidades de desarrollo] Extraído el 5 de mayo, de 2007 de www.albany.edu/aging/IDD/ documents/parentworkbook.pdf

Better Health Channel. (2004). Sex education for children with intellectual disabilities [La educación sexual para niños con discapacidad intelectual]. Extraído el 10 de marzo, de 2007 de http://www.disability.vic. gov.au/dsonline/dsarticles.nsf/pages/ Sex_education_for_children_with_ intellectual_disabilities
Better Health Channel (2007). "Sex education for children with intellectual disabilities: tips for parents" [Educación Sexual para niños con discapacidad intelectual: consejos para padres]. Recuperado el 10 de marzo, 2007 en

http://www.betterhealth.vic.gov. au/BHCV2/bhcarticles.nsf/pages/ Sex_education_for_children_with intellectual_disabilities_tips_for parents?OpenDocument

Código de la Niñez y la Adolescencia. (s.f.). Título II. Derechos y Obligaciones. Capítulo IV. Derecho a la Salud. Artículo 55- Consultado el 10 de marzo, 2007 de

www.poder-judicial.go.cr/ salasegunda/normativa/Código\%20 de $\% 201 \mathrm{l} \% 20 \mathrm{Niñez} \% 20 \mathrm{y} \% 20 \mathrm{la} \% 20$

Adolescencia.doc

Consejo Nacional de Rehabilitación y Educación Especial. "¿Quién es el CNREE?”. Recuperado el 25 de mayo, 2007 de http://www.redespecialcr.net/ Principal_CentroApoyo.html

Convención de los derechos de las personas con discapacidad (2007). Recuperado el 10 de junio de 2007 de www.un.org/ esa/socdev/enable

Enciclopedia Libre Universal en Español. (s.f.). Mito. Extraída el 25, junio, de 2007 de www.enciclopedia.us.es/wiki. phtml?tittle=Mito

Ministerio de Educación Pública [MEP]. (2001). Políticas de Educación Integral de la Expresión de la Sexualidad Humana. San José, Costa Rica: s.e.

Ministerio de Educación Pública [MEP]. (2007). Conceptos básicos de sexualidad. Trabajo presentado en la Capacitación Educación de la 
Sexualidad para Multiplicadores. [Diapositivas en Power Point]. San José, Costa Rica.

Ministerio de Salud. Organización Panamericana para la Salud. (2004). La discapacidad en Costa Rica: situación actual y perspectivas. Extraído el 10 de abril, 2007 de

http://www.196.40.24.244/php/ situacion/discapacidad.pdf

Murillo, A y Richmond, V. (2005). La educación de la sexualidad en las personas que presentan discapacidad. Manuscrito no publicado, sin paginación.

Oficina de Alto Comisionado para los Derechos Humanos. (1990). Convención sobre los derechos del niño. Extraído el 10 de junio, de 2007 de www.unhchr.ch/spanish/html/ menu3/b/k2crc_sp.htm

Silverberg, C. (2006). Sexuality and Disability Myths and Facts
[Sexualidad, Discapacidad Mitos y Hechos]. Extraído el 10 de marzo, de 2007 en

www.sexuality.about.com/od/

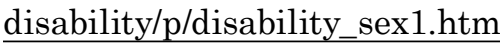

Ruiz, X. (1999, Enero-Junio). Pedagogía de la sexualidad. Tiempo de educar. Revista interinstitucional de Investigación Educativa, 001(1), 119. 130. Extraído el 10 de marzo, de 2007 de

http://redalyc.uaemex.mx/redalyc/src/ inicio/ArtPdfRed.jsp?iCve=31100107

The National Information Center for Children and Youth with Disabilities. (1992). Sexuality education for children and youth with disabilities [Educación sexual para niños y jóvenes con discapacidad]. Recuperado el 10 de marzo, de 2007 de

www.nichcy.org/pubs/outprint/ nd17txt.htm 